\title{
Ascending Stairway Modeling: \\ A First Step Toward Autonomous Multi-Floor Exploration
}

\author{
Jeffrey A. Delmerico, Jason J. Corso, David Baran, Philip David, and Julian Ryde
}

\begin{abstract}
Many robotics platforms are capable of ascending stairways, but all existing approaches for autonomous stair climbing use stairway detection as a trigger for immediate traversal. In the broader context of autonomous exploration, the ability to travel between floors of a building should be compatible with path planning, such that the robot can traverse a stairway at a time that is appropriate to its navigation goals. No system yet presented is capable of both localizing stairways on a map and estimating their properties, functions that in combination would enable stairways to be considered as traversable terrain in a path planning algorithm. We propose a method for modeling stairways as objects and localizing them on a map, such that they can be subsequently traversed if they are of dimensions that the robotic platform is capable of climbing. Our system consists of two parts: a computationally efficient detector that leverages geometric cues from depth imagery to detect sets of ascending stairs, and a stairway modeler that uses multiple detections to infer the location and parameters of a stairway that is discovered during exploration. This video demonstrates the performance of the system in a number of real-world situations, modeling and localizing a variety of stairway types in both indoor and outdoor environments.
\end{abstract}

\section{INTRODUCTION}

When exploring a new space, humans typically investigate the places that are easy to get to first, and only later move into less accessible areas. In the context of a multi-floor building, this translates to people fully exploring the ground floor of the building before traveling via stairs or elevators to other floors that require more effort to reach. A logical approach to autonomous exploration in a multi-floor building would also follow a similar strategy: explore the accessible areas on the current floor before undertaking the more difficult task of transitioning to another floor to find less accessible areas. Existing approaches for stair detection serve to initiate their traversal as soon as they are detected, which is not compatible with the aforementioned approach to building exploration. None of these methods have been incorporated into a comprehensive multi-floor exploration approach.

The restriction of robots to single floors of a building or outdoor areas free of abrupt elevation changes presents a significant limitation to real-world applications, such as whole-building mapping and rescue scenarios. Our work seeks a solution to this problem and is motivated by the rich potential of an autonomous ground robot that can climb stairs while exploring a multi-floor building. Our proposed solution to this problem is a system to detect and localize stairways in the environment during the process of exploration, and

J. Delmerico, J. Corso, and J. Ryde are with SUNY Buffalo \{jad12, jcorso, jryde\}abuffalo.edu

D. Baran and P. David are with the U.S. Army Research Laboratory \{david.g.baran.civ, philip.j.david4.civ\}@mail.mil

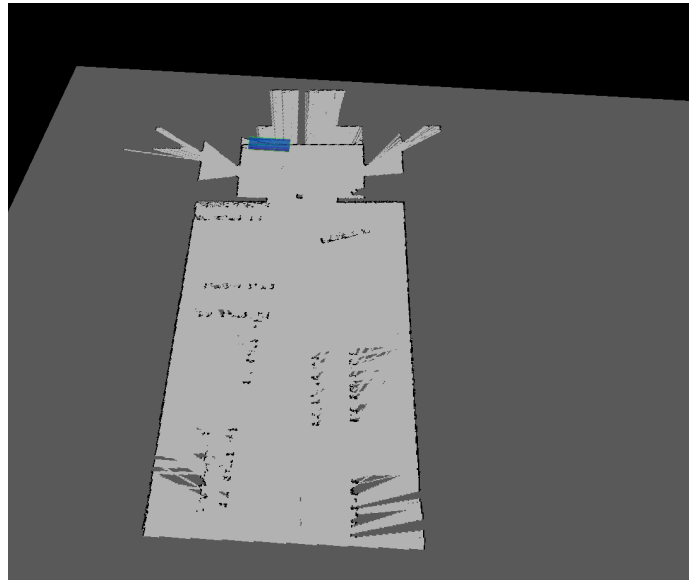

Fig. 1. Occupancy grid map with detected and modeled stairway superimposed (upper left).

model any identified stairways in order to determine if they are traversable by the robot. With a map of the environment and estimated locations and parameters of the stairways, the robot could plan a path that traverses the stairs in order to explore the frontier at other elevations that were previously inaccessible. Autonomous multi-floor exploration is a new behavior for ground robots, and we present this video as a summary of our first steps toward the realization of that capability.

\section{A. Background}

Other systems have been proposed for related tasks, but no existing work approaches the problem in the context of the aforementioned scenario. Bansal et al. [1] detect and localize stairways while constructing a map, but do not assess traverability. Several methods [2], [3], [4] perform detection and traversal, but do not model the pose or location of the stairway and and simply, immediately initiate a climbing mechanism. Although these capabilities are related to our problem scenario, immediate climbing is not necessarily compatible with a mapping task. Path planning for multifloor exploration should take the stairway into account as a portal to more unexplored regions, but traversing stairs immediately upon a single detection makes exploring the low-cost frontiers of the original floor more difficult and may fail if the detection was erroneous.

\section{B. Methods}

We employ a computationally lightweight stair edge detector that operates by finding lines of range discontinuity in depth imagery (here produced by a Microsoft Kinect sensor) 

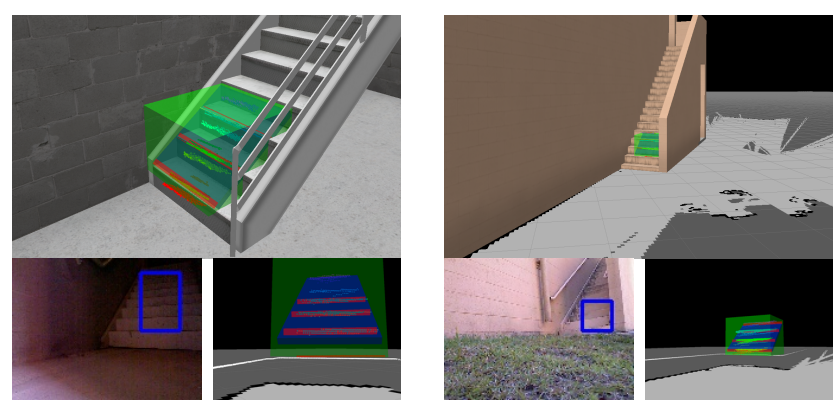

Fig. 2. Results of several runs from our datasets showing the stairway model superimposed on a rendered building model, a marked-up image showing a detection, and a view of the map and model from the robot's perspective.

that match the signature of sets of stairs: vertically clustered sets of nearly parallel lines. Point clouds corresponding to the detected stair edges are extracted and aggregated together, with approximate alignment provided by the robot's estimated pose from mapping. To this aggregated point cloud we fit a generative stairway model, and we assume a nonspiral staircase. In testing, we have observed that the system is capable of detecting stairways with non-parallel steps, for example a stairway with a $90^{\circ}$ turn over several steps. However, many ground robots are not capable of traversing tight spiral stairs, and so we do not focus on these types.

The stairway is represented as a single object: an inclined plane constrained by a bounding box, with parallel stair edges lying in the plane. This model is parameterized by the bounding box centroid $\left(B_{x}, B_{y}, B_{z}\right)$ and dimensions $(H, W, D)$, pitch relative to the ground plane $(P)$, and step dimensions $(h, d)$. We assume that stair steps are approximately parallel to the ground plane, so the bounding box top and bottom are parallel to the $X Y$ plane. For an inclined plane model of:

$$
a x+b y+c z+d=0
$$

the planes constituting the bounding box are given by:

$$
\begin{gathered}
z=B_{z} \pm \frac{H}{2} \\
a\left(x-B_{x}\right)+b\left(y-B_{y}\right) \pm \frac{D}{2}\left(\sqrt{a^{2}+b^{2}}\right)=0 \\
-c b\left(x-B_{x}\right)+c a\left(y-B_{y}\right) \pm \frac{W}{2}\left(c \sqrt{a^{2}+b^{2}}\right)=0
\end{gathered}
$$

for the top/bottom, front/back, and sides, respectively. Here, the pitch $P$ is computed as the dihedral angle of the planar model and the ground plane: $P=\arccos (a, b, c) \cdot(0,0,1)$. As new observations are added to the aggregated point cloud, the model parameters are re-estimated, outliers are removed, and well-supported stair edges are used to infer the dimensions of each step.

Work on a comprehensive system for multi-floor exploration is ongoing. Current investigations include developing techniques for robustly discovering all stairways in the environment and path planning for multi-floor exploration.

\section{RESUlts}

Our video sequences show the evolution of the model over multiple observations, the refinement of the parameter
TABLE I

TABLE OF MODEL STEP ESTIMATES AND GROUND TRUTH VALUES (GT)

\begin{tabular}{|c|c|c|c|c|}
\hline Trial & Height $(\mathbf{m})$ & Depth $(\mathbf{m})$ & Width $(\mathbf{m})$ & Pitch $\left(^{\circ}\right)$ \\
\hline B.1 Front & 0.174 & 0.257 & 0.977 & 34.0 \\
GT & $\mathbf{0 . 1 9 6}$ & $\mathbf{0 . 2 5 4}$ & $\mathbf{0 . 9 6 5}$ & $\mathbf{3 7 . 6}$ \\
\hline B.1 Rear & 0.166 & 0.252 & 0.715 & 33.0 \\
GT & $\mathbf{0 . 1 9 6}$ & $\mathbf{0 . 2 5 4}$ & $\mathbf{0 . 9 6 5}$ & $\mathbf{3 7 . 6}$ \\
\hline B.3 & 0.169 & 0.245 & 0.689 & 35.4 \\
GT & $\mathbf{0 . 1 9 2}$ & $\mathbf{0 . 2 6 3}$ & $\mathbf{1 . 0 1 5}$ & $\mathbf{3 6 . 2}$ \\
\hline Davis Front & 0.175 & 0.323 & 1.076 & 28.3 \\
GT & $\mathbf{0 . 1 8 1}$ & $\mathbf{0 . 3 0 5}$ & $\mathbf{1 . 2 2 6}$ & $\mathbf{3 0 . 7}$ \\
\hline Davis Rear & 0.169 & 0.311 & 1.048 & 29.4 \\
GT & $\mathbf{0 . 1 6 5}$ & $\mathbf{0 . 2 9 2}$ & $\mathbf{1 . 1 7 5}$ & $\mathbf{2 9 . 5}$ \\
\hline Mean Error & 0.017 & 0.012 & 0.173 & 2.3 \\
\hline Std. Dev. & 0.01396 & 0.01568 & 0.1285 & 1.876 \\
\hline
\end{tabular}

estimates with each model fitting, and the accuracy of stairway localization within the map. It should be noted that in the video sequences as well as the image stills in Fig. 2, 3D meshes were available for some of the buildings being observed, and in our visualizations, those models were aligned with the underlying map, and not with the stairway model.

We can accurately localize a detected and modeled stairway in a large map (See Fig. 1), and over many groundtruthed trials, this system has proven to be accurate in the estimation of the stairway model's parameters (See Table I). It is robust to false positive detections, since they are removed as statistical outliers in the modeling phase. However, our detector has also proven to be highly accurate, with only a handful of false positive frames over many hours of testing. Despite the limitations of structured light sensors such as the Microsoft Kinect in outdoor environments, we found that in many circumstances even the compromised depth data in outdoor settings was adequate to build an accurate model. In all of our experiments mapping realworld environments containing stairways, this system was able to produce an accurately localized model with sufficient parameter estimates for the robot to determine whether the stairway is traversable (See Fig. 2). Thus, in an autonomous exploration scenario, stairways in the environment can be localized during the process of mapping the initial floor of a building, which represents a first step toward exploration of buildings with multiple floors.

\section{REFERENCES}

[1] M. Bansal, B. Matei, B. Southall, J. Eledath, and H. Sawhney, "A lidar streaming architecture for mobile robotics with application to $3 \mathrm{~d}$ structure characterization," in Robotics and Automation (ICRA), 2011 IEEE International Conference on, may 2011, pp. $1803-1810$.

[2] A. M. Johnson, M. T. Hale, G. C. Haynes, and D. E. Koditschek, "Autonomous legged hill and stairwell ascent," in IEEE International Workshop on Safety, Security, \& Rescue Robotics (SSRR), November 2011, pp. 134-142.

[3] E. Mihankhah, A. Kalantari, E. Aboosaeedan, H. Taghirad, S. Ali, and A. Moosavian, "Autonomous staircase detection and stair climbing for a tracked mobile robot using fuzzy controller," in Proceedings of the IEEE International Conference on Robotics and Biomimetics. IEEE, 2008, pp. 1980-1985.

[4] R. Ray, B. Bepari, and S. Bhaumik, "On design and development of an intelligent mobile robotic vehicle for stair-case navigation," in Intelligent Autonomous Systems. Springer, 2010, pp. 87-122. 